\title{
¿El deterioro cognitivo en los primeros episodios de esquizofrenia es generalizado o selectivo?
}

\author{
Gemma Garrido García ${ }^{1}$ y Joan Alberni Coderch ${ }^{2}$ \\ ${ }^{1}$ Unidad de Hospitalización Parcial de Salud Mental. Ámbito de Atención a la Salud Mental. Consorci Sanitari de Terrassa \\ (Barcelona). \\ 2Dirección científica. Ámbito de Atención a la Salud Mental. Consorci Sanitari de Terrassa (Barcelona).
}

\section{Resumen}

El desarrollo de las neurociencias ha evidenciado que la esquizofrenia tiene una base neurobiológica clara y que la alteración neuropsicológica es un aspecto central de esta enfermedad que interfiere directamente en la adaptación funcional y en la adherencia al tratamiento de estos pacientes. Un aspecto a tener en cuenta es que la disfunción cognitiva precede a los síntomas psicóticos; además, tras la estabilidad clínica, la alteración neuropsicológica se mantiene estable o incluso puede progresar a lo largo de la enfermedad. A pesar de los esfuerzos en investigación, en la actualidad se sigue debatiendo si el deterioro de estos enfermos es global o selectivo durante las primeras etapas del trastorno. El objetivo de este trabajo es revisar los dominios de la función cognitiva que han ofrecido mayor controversia en los primeros episodios de la enfermedad: a) funcionamiento intelectual general; b) memoria; c) función ejecutiva, y d) velocidad de procesamiento.

(Alzheimer. Real Invest Demenc. $2011 ; 48: 18-24)$

Palabras clave: esquizofrenia, primeros episodios, cognición, neuropsicología, inteligencia.

\section{Abstract}

The development of neuroscience has proved that schizophrenia has a clear neurobiological basis and that neuropsychological disfunction is a central feature of schizophrenia that interferes directly in the functional adaptation and medical adherence of patients with this illness. An aspect to take into account is that cognitive disfunction precedes psychotic symptoms and that after clinical stability, the impaired cognition remains stable or even progresses in the course of illness. Regardless the efforts of investigation, currently if global or selective impairment cognition in the first episode of the illness takes place, debates continue emerging. The aim of this article is to offer a review of the domains of the cognitive function that have shown controversy in the first episode of schizophrenia: a) intellectual ability; b) memory; c) executive function, and d) speed of processing.

(Alzheimer. Real Invest Demenc. 2011;48:18-24)

Keywords: schizophrenia, first-episode, cognition, neuropsychology, intelligence.

\section{Introducción}

El término esquizofrenia hace referencia a una enfermedad crónica que se caracteriza por unas dimensio-

Recibido para su publicación: 23 de julio de 2010. Aceptado para su publicación: 8 de septiembre de 2010. Correspondencia: G. Garrido García.

E-mail: ggarrido@cst.cat nes sintomáticas (síntomas negativos y positivos, déficits cognitivos) con una base neurobiológica clara ${ }^{1,2}$.

Kraepelin aludía a la pérdida de funciones o capacidades intelectuales y denominaba a esta enfermedad demencia precoz; de esta forma señalaba el deterioro cognitivo como una característica importante del trastorno. Pero no ha sido hasta el desarrollo de las neurociencias cuando se ha podido concebir como una patología cerebral ${ }^{3,1}$ en que la disfunción cognitiva afec- 
ta a la mayoría de los pacientes, por lo que ha sido considerada por algunos autores como un signo patognómico de la enfermedad ${ }^{4}$. La investigación en las últimas décadas ha evidenciado que el rendimiento de las personas con esta enfermedad es inferior al de los sujetos sanos en diferentes tipos de dominios cognitivos: capacidad intelectual, atención, memoria, capacidad ejecutiva y velocidad de procesamiento ${ }^{5}$. Esto repercute directamente en la adaptación funcional ${ }^{6,7}$ y en la adherencia al tratamiento ${ }^{8}$. Un aspecto a tener en cuenta es que la alteración cognitiva está presente en los estadios premórbidos ${ }^{9}$ y en los primeros episodios de la enfermedad ${ }^{10}$, pero actualmente se sigue debatiendo si el deterioro de estos enfermos es global o selectivo.

El objetivo de este trabajo es revisar aquellos aspectos de la función cognitiva en los que ha habido mayor controversia en los primeros episodios de la enfermedad para poder comprender qué dimensiones de riesgo están presentes en las primeras etapas del trastorno.

\section{Funcionamiento intelectual general}

La hipótesis del neurodesarrollo temprano concibe la esquizofrenia como un trastorno en la conectividad neural durante el período prenatal por causas genéticas o de daño perinatal que interaccionaría en el proceso de maduración normal y produciría anormalidades cerebrales como las que se describen en algunas publicaciones $^{11,12}$.

Una de las dimensiones que mejor pueden reflejar la alteración en el neurodesarrollo temprano es el funcionamiento intelectual general o coeficiente intelectual (CI) premórbido evaluado mediante escalas de inteligencia. Estudios centrados en el CI sugieren que los individuos con esquizofrenia obtienen niveles premórbidos inferiores respecto al grupo control ${ }^{13-15,10}$. Sin embargo, otras publicaciones informan de un rendimiento normal, semejante al grupo control ${ }^{16}$, exceptuando algunas tareas ${ }^{17}$.

El hecho de que existan pacientes con un CI normal y con unas habilidades alteradas desafía el concepto de inteligencia general de Spearman como factor que subyace a las habilidades para la realización de dife- rentes tipos de tareas. Algunos autores sugieren que los pacientes con CI normal pueden ser un discreto subgrupo con un menor daño en el neurodesarrollo ${ }^{18}$, lo que enfatiza la teoría de reserva cognitiva ${ }^{19}$ como la capacidad que tienen los pacientes de compensar la disfunción cerebral ${ }^{20}$.

\section{Función ejecutiva}

El concepto de función ejecutiva engloba una cantidad de habilidades cognitivas como planificar, iniciar, secuenciar, verificar e inhibir una conducta para conseguir un objetivo. Este tipo de función está claramente alterada en los pacientes con esquizofrenia desde los primeros episodios de la enfermedad en la población no medicada ${ }^{21}$ y en las personas con un alto riesgo de desarrollar el trastorno ${ }^{22}$. Este dominio cognitivo se ha fraccionado en diferentes componentes como la memoria de trabajo (MT), habilidad de cambio e inhibición conductual.

La MT, un concepto propuesto por Baddeley, es el modo de operar o trabajar de la memoria a corto plazo. Este autor propuso inicialmente tres subcomponentes: el central ejecutivo, que es un sistema de control atencional; y dos subsistemas encadenados, el dispositivo visoespacial, que permite manipular las imágenes visuales, y el retén fonológico, que almacena y repasa la información basada en el lenguaje. Así, el central ejecutivo y sus subsistemas nos permiten mantener y manejar temporalmente la información, comprender el lenguaje, efectuar cálculos, razonar, aprender y solucionar problemas. Posteriomente, se añade un cuarto componente: el buffer episódico de capacidad limitada, controlado por el central ejecutivo, el cual accede a la información contenida mediante procesos de atención consciente, que integra información episódica ${ }^{2,3}$ (fig. 1).

Los déficits en la MT están presentes en pacientes no medicados ${ }^{24}$, incluso en aquellos con un mayor CI, que presentan una alteración específica en la $\mathrm{MT}^{25}$ en estudios centrados en el período prodrómico de la enfermedad $^{26}$. Estudios de metaanálisis centrados en los primeros episodios muestran que esta alteración ya está 


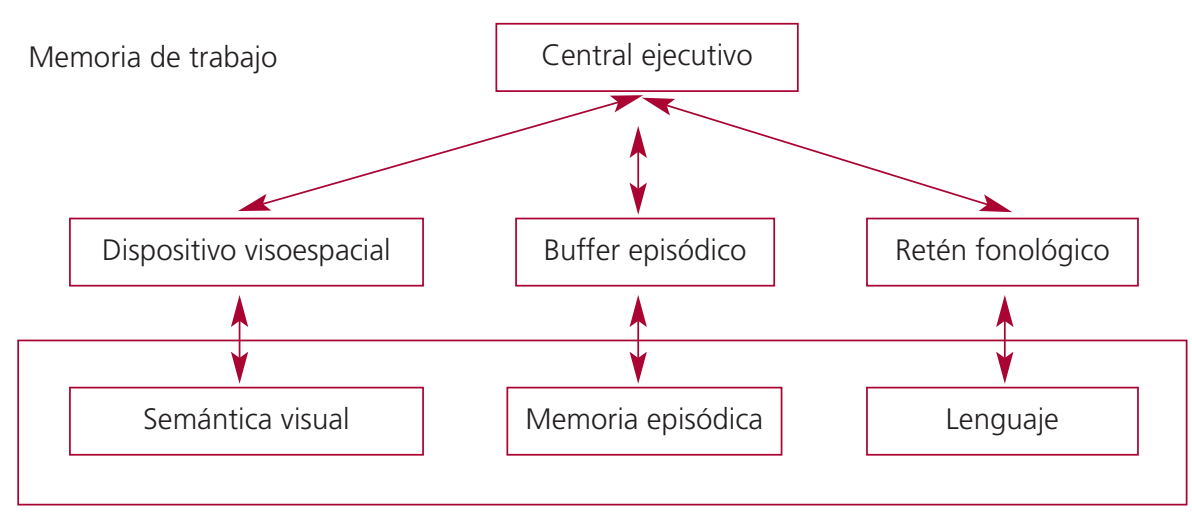

Figura 1. Modelo multicomponente de la memoria de trabajo de Baddeley ${ }^{23}$.

presente pero que no es superior a otros dominios cognitivos ${ }^{10}$.

En cambio, otros autores, que comparan población con riesgo de psicosis con pacientes en el primer episodio y controles sanos, detectan un peor rendimiento en la MT en los dos primeros grupos en comparación con los sujetos sanos. Además, cuando se analiza longitudinalmente el grupo de los pacientes de riesgo que han padecido un primer episodio, éstos muestran un peor rendimiento tanto en la MT como en la velocidad de procesamiento, mientras que los que no han evolucionado hacia la psicosis se mantienen estables en su rendimiento ${ }^{27}$.

El test de clasificación de cartas de Wisconsin (WCST, del inglés Wisconsin Card Sorting Test) es el instrumento más utilizado como medida general de función ejecutiva en este tipo de pacientes. Los fracasos detectados sugieren dificultades a la hora de pensar en términos abstractos y una incapacidad para cambiar la actuación de acuerdo con un resultado, a pesar de comprender bien las normas e instrucciones; tienen dificultades para usar la información que han obtenido del medio a fin de cambiar su conducta.

Mesholam-Gately ${ }^{10}$, en su estudio de metaanálisis, analiza 47 investigaciones sobre medidas neurocognitivas en pacientes en el primer episodio de la enfermedad y concluye que la alteración cognitiva se muestra en diez dominios cognitivos diferentes. Concretamente, la alteración en la función ejecutiva evaluada con diferentes índices del WCST mostraba gran variedad entre sí, lo que evidencia que se trata de un dominio complejo formado por diferentes subcomponentes. Así lo demuestran estudios de neuroimagen funcional, que evidencian que el WCST no es una medida específica y manifiestan un patrón diferencial de activación según las demandas: la corteza prefrontal $(\mathrm{CPF})$ ventrolateral derecha en operaciones simples de MT y la CPF dorsolateral derecha en aquellas tareas que requerían una mayor demanda de MT. La activación entre la zona temporoparietal y la zona rostral de la corteza cingulada anterior se asociaba con la detección de errores, mientras que la activación entre la corteza cingulada anterior caudal y la CPF dorsolateral derecha se vinculaba con las mayores demandas de la memoria operativa y el control atencional ${ }^{28}$.

\section{Memoria}

Uno de los dominios neuropsicológicos que más se ha asociado a la esquizofrenia es la alteración de la memoria. Para algunos autores, es una de las medidas más útiles para predecir la transición hacia la psicosis que se ha descrito en todas las fases de la enfermedad. Como la memoria no es una función unitaria y se han conceptualizado diferentes tipos de ella, cabe pregun- 
tarse si existe un tipo de memoria más afectado en este trastorno y concretamente en relación con los primeros episodios.

El trastorno en la memoria episódica es más relevante que los déficits de las memorias implícita y semántica en estas etapas de la enfermedad. Concretamente, se han detectado déficits en la memoria verbal en las primeras etapas de la enfermedad ${ }^{29}$. Los déficits en el proceso de codificación son los más descritos, y no queda claro si el proceso de retención del material verbal está alterado. Así, para algunos autores los trastornos de la memoria en la esquizofrenia se deberían a problemas en la codificación y recuperación de la información a largo plazo, de modo que estos pacientes mostrarían dificultades a la hora de registrar la información y los recuerdos se evocarían con dificultad.

Si la alteración en la codificación y en el recuerdo se asocia a la CPF y, además, implica habilidad para planificar, crear estrategias e inhibir la distracción ${ }^{30}$, ¿estas alteraciones son independientes de la alteración en la función ejecutiva?

Estudios centrados en los primeros episodios sugieren que los déficits en aprendizaje verbal, función ejecutiva y CI pueden reflejar una alteración en el procesamiento de la información en la $\mathrm{CPF}^{31}$. Los estudios de metaanálisis en neuroimagen funcional informan de una alteración en la actividad de la CPF en el grupo experimental al realizar tareas de MT, y en la prueba WCST ${ }^{32}$.

\section{Velocidad de procesamiento}

Las tareas que evalúan la velocidad del procesamiento de la información (VPI) incluyen la integridad de diferentes componentes cognitivos. Como propuso Solthouse en 1996, el enlentecimiento del procesamiento de la información es responsable de un peor rendimiento en las tareas que se han de realizar en un tiempo determinado (mecanismo de tiempo limitado) y en aquellas en que el propio enlentecimiento reduce la cantidad de información necesaria para un mayor nivel de procesamiento (mecanismo de simultaneidad). Así, los déficits en la VPI podrían emerger por la discrepancia entre la pérdida de información en el transcurso del tiempo y la velocidad con la que determinadas operaciones deben ejecutarse con éxito, como la codificación, la elaboración, la búsqueda, la abstracción y la integración de la información.

De las diferentes pruebas que se han empleado para evaluar este dominio cognitivo, la tarea de codificación de la clave de números $(\mathrm{CN})$ de la Escala de inteligencia para adultos de Wechsler es una de las más utilizadas. En un estudio de metaanálisis, se demostró un mayor efecto de tamaño para esta tarea en comparación con otros dominios cognitivos ${ }^{33}$. Este rasgo de la enfermedad también se ha considerado importante en estudios de metaanálisis centrados en primeros episodios ${ }^{10}$. Estudios de primeros episodios de esquizofrenia, que comparan pacientes con deterioro cognitivo, pacientes sin deterioro cognitivo y sujetos sanos, muestran una clara diferencia entre el grupo de pacientes y el grupo de control en la tarea de velocidad de procesamiento; además, se mantiene ligeramente esta diferencia entre el grupo de pacientes sin deterioro cognitivo y los controles. Otro dato importante a tener en cuenta en esta investigación es que el grupo con deterioro cognitivo tenían un número menor de años de educación, y menor CI premórbido en comparación con el grupo de pacientes sanos y los controles ${ }^{34}$.

La hipótesis de que el enlentecimiento en el procesamiento de la información determina el deterioro en diferentes dimensiones cognitivas, al reducir la habilidad para integrar y organizar la información de forma eficiente, se ha demostrado en diferentes publicaciones con pacientes crónicos ${ }^{35}$, y se ha sugerido su papel como rasgo principal en los primeros estadios de la enfermedad ${ }^{36}$.

Así, siguiendo el modelo de Salthousen, cuando se investiga detalladamente el rendimiento en tareas de CI mediante la Escala de inteligencia para adultos de Wechsler en muestras de pacientes en las primera etapas de la enfermedad y controles sanos emparejados según los índices de la batería, se halla que los pacientes rinden mejor en medidas de comprensión verbal y organización perceptiva, y peor en los factores de velocidad de proceso y MT. Concretamente, el subtest de CN mostraba un mayor efecto de tamaño discriminando entre pacientes y sujetos sanos ${ }^{4}$. 
Estos resultados son compatibles con estudios que demuestran que pacientes con una función intelectual normal muestran un rendimiento alterado en medidas de velocidad de procesamiento y en tareas de tiempo de inspección ${ }^{37}$ y con otras investigaciones que detectan correlaciones significativas entre el rendimiento de la velocidad de procesamiento y medidas de memoria y función ejecutiva ${ }^{38}$.

En un estudio prospectivo en el que se evalúa el funcionamiento cognitivo en la infancia de individuos que posteriormente desarrollan la enfermedad de la esquizofrenia, en comparación con sus hermanos no afectados y un grupo de control, se halló que el grupo de los afectados tienen un rendimiento significativamente inferior en la prueba de $\mathrm{CN}^{39}$.

En un reciente estudio, en el que se comparan pacientes en el primer episodio de la enfermedad y el grupo de control, emparejados por edad, sexo y CI, se muestra que el rendimiento en la velocidad de proceso es significativamente inferior en el grupo de pacientes. Al volver a testar ambas muestras un año después, el grupo control mejoró significativamente, mientras que el grupo de los pacientes no mostró cambios en su rendimiento, a pesar de la mejora clínica tras el tratamiento farmacológico, lo que evidencia que este dominio cognitivo es un posible marcador para la enfermedad, que contribuye de forma significativa a la alteración de otras funciones como la memoria episódica y, especialmente, la $\mathrm{MT}^{40}$.

\section{Conclusión}

La disfunción cognitiva es un aspecto importante de la enfermedad de la esquizofrenia que determina la adaptación funcional y el pronóstico de las personas que la padecen.

La alteración neuropsicológica se detecta ya en los primeros episodios del trastorno y en estadios premórbidos. Si bien parece que el deterioro de la enfermedad es global, en las primeras etapas del trastorno parece ser más selectivo.

La VPI requiere de la integridad de diferentes componentes cognitivos, lo que hace que el enlentecimiento repercuta en la habilidad para mantener la información, organizar e integrar.

De esta manera, los dominios más susceptibles a esta alteración son la función ejecutiva, especialmente la MT, el proceso de codificación y recuerdo a largo plazo en la memoria episódica.

Estudios centrados en primeros episodios de la enfermedad demuestran que los pacientes rinden peor en tareas de VPI; se ha demostrado en estudios de metaanálisis un mayor efecto de tamaño en este dominio cognitivo, se ha propuesto como un índice que discrimina entre el grupo experimental y el control que se halla presente en etapas premórbidas y se ha sugerido como una dimensión de riesgo.

Diferentes estudios reflejan que estos pacientes pueden presentar alteraciones en otros dominios que son dependientes de los mecanismos de tiempo limitado y simultaneidad como son el CI, la función ejecutiva, y la memoria en su proceso de codificación y recuerdo a largo plazo.

Los pacientes con un CI equiparable al grupo de control pueden ser un discreto subgrupo con un menor daño en el neurodesarrollo o que posee mayor reserva cognitiva para hacer frente al trastorno.

La información derivada de estos estudios es de gran utilidad clínica, ya que ayuda a plantear intervenciones farmacológicas y cognitivas más adaptadas a los primeros estadios de la enfermedad para poder mejorar los procesos de rehabilitación de las personas que la padecen.

En definitiva, la investigación en las primeras etapas del trastorno permite esclarecer los mecanismos subyacentes a la enfermedad, los rasgos que predisponen hacia el trastorno (neurobiológicos y neuropsicológi$\cos )$ y, de esta manera, se pueden realizar intervenciones tempranas más válidas y específicas.

\section{Bibliografia}

1. Austin J. Schizophrenia: an update and review. J Genet Couns. 2005 Oct; 14(5):329-40.

2. Wong AH, Van Tol HH. Schizophrenia: from phenomenology to neurobiology. Neurosci Biobehav Rev. 2003 May; 27(3):269-306. 
3. Heinrichs RW.The primacy of cognition in schizophrenia. Am Psychol. 2005 Apr;60(3):229-42.

4. Wilk CM, Gold JM, McMahon RP, Humber K, Iannone VN, Buchanan RW. No, it is not possible to be schizophrenic yet neuropsychologically normal. Neuropsychology. 2005 Nov; 19(6):778-86.

5. Heinrichs RW, Zakzanis KK. Neurocognitive deficit in schizophrenia: a quantitative review of the evidence. Neuropsychology. 1998 Jul;12(3):426-45.

6. Green MF. What are the functional consequences of neurocognitive deficits in schizophrenia?Am J Psychiatry. 1996 Mar;153(3):321-30.

7. Green MF, Kern RS, Braff, DL Mintz, J. Neurocognitive deficits and functional outcome in schizophrenia: are we measuring the right stuff? Schizophrenia Bulletin. 2000; 26:119-36.

8. Maeda K, Kasai K, Watanabe A, Henomatsu K, Rogers MA, Kato N.Effect of subjective reasoning and neurocognition on medication adherence for persons with schizophrenia. Psychiatr Serv. 2006 Aug;57(8):1203-5.

9. Keefe RS, Perkins DO, Gu H, Zipursky RB, Christensen BK, Lieberman JA. A longitudinal study of neurocognitive function in individuals at-risk for psychosis. Schizophr Res. 2006 Dec;88(1-3):26-35. Epub 2006 Aug 22.

10. Mesholam-Gately RI, Giuliano AJ, Goff KP, Faraone SV, Seidman LJ. Neurocognition in first-episode schizophrenia: a meta-analytic review. Neuropsychology. 2009 May; 23(3):315-36.

11. Weinberger DR. Implications of normal brain development for the pathogenesis of schizophrenia. Arch Gen Psychiatry. 1987 Jul;44(7):660-9.

12. Vita A, De Pieri L, Silenzi C, Dieci M. Brain morphology in first-episode schizophrenia: a meta-analysis of quantitative magnetic resonance studies. Schizophrenia Research. 2006 Feb 15;82(1):75-88.

13. Aylward E, Walker E, Bettes B. Intelligence in schizophrenia: meta-analysis of the research. Schizophr Bull. 1984; 10(3):430-59.

14. Woodberry KA, Giuliano AJ, Seidman LJ. Premorbid IQ in schizophrenia: a meta-analytic review. Am J Psychiatry. 2008 May;165(5):579-87. Epub 2008 Apr 15.

15. Sorensen HJ, Mortensen EL, Schiffman J, Ekstrøm M, Denenney D, Mednick SA. Premorbid IQ and adult schizophrenia spectrum disorder: verbal performance subtests. Psychiatry Res. 2010 May 5:23-6.

16. Palmer BW, Heaton RK, Paulsen JS, Kuck J, Braff D, Harris MJ, et al. Is it possible to be schizophrenic yet neuropsychologically normal? Neuropsychology. 1997 Jul; $11(3): 437-46$

17. González-Blanch C, Rodríguez-Sánchez JM, PérezIglesias R, Pardo-García G, Martínez-García O, VázquezBarquero JL, et al. First-episode schizophrenia patients neuropsychologically within the normal limits: evidence of deterioration in speed of processing. Schizophr Res. 2010 Jun;119(1-3):18-26. Epub 2010 Mar 23.

18. MacCabe JH, Aldouri E, Fahy TA, Sham PC, Murray RM. Do schizophrenic patients who managed to get to university have a non-developmental form of illness? Psychol Med. 2002 Apr;32(3):535-44.

19. Satz P, Morgenstern H, Miller EN, Selnes OA, McArthur JC, Cohen BA, et al. Low education as a possible risk factor for cognitive abnormalities in HIV-1: findings from the multicenter AIDS Cohort Study (MACS). J Acquir Immune Defic Syndr. 1993 May;6(5):503-11.

20. Holthausen EA, Wiersma D, Sitskoorn MM, Hijman R, Dingemans PM, Schene AH, et al. Schizophrenic patients without neuropsychological deficits: subgroup, disease severity or cognitive compensation? Psychiatry Res. 2002 Sep 15;112(1):1-11.

21. Chan RC, Chen EY, Law CW. Specific executive dysfunction in patients with first-episode medication-naïve schizophrenia. Schizophr Res. 2006 Feb 15;82(1):51-64. Epub 2005 Dec 2.

22. Smith CW, Park S, Cornblatt B. Spatial working memory deficits in adolescents at clinical high risk for schizophrenia. Schizophr Res. 2006 Jan 31;81(2-3):211-5.

23. Repovs G, Baddeley A.The multi-component model of working memory: explorations in experimental cognitive psychology. Neuroscience. 2006 Apr 28;139(1):5-21. Epub 2006 Mar 6.

24. Carter C, Robertson L, Nordahl T, Chaderjian M, Kraft L, O'Shora-Celaya L. Biol Spatial working memory deficits and their relationship to negative symptoms in unmedicated schizophrenia patients. Psychiatry. 1996 Nov 1; 40(9):930-2.

25. Joyce EM, Hutton SB, Mutsatsa SH, Barnes TR. Cognitive heterogeneity in first-episode schizophrenia. Br J Psychiatry. 2005 Dec;187:516-22.

26. Hawkins KA, Addington J, Keefe RS, Christensen B, Perkins DO, Zipurksy R, et al. Neuropsychological status of subjects at high risk for a first episode of psychosis. Schizophr Res. 2004 Apr 1;67(2-3):115-22.

27. Jahshan C, Heaton RK, Golshan S, Cadenhead KS. Course of neurocognitive deficits in the prodrome and first episode of schizophrenia. Neuropsychology. 2010 Jan;24(1):109-20.

28. Lie CH, Specht K, Marshall JC, Fink GR.Using fMRI to decompose the neural processes underlying the Wisconsin Card Sorting Test. Neuroimage. 2006 Apr 15;30(3):1038-49. Epub 2006 Jan 18.

29. Eastvold AD, Heaton RK, Cadenhead KS.Neurocognitive deficits in the (putative) prodrome and first episode of psychosis. Schizophr Res. 2007 Jul;93(1-3):266-77. Epub 2007 Apr 30.

30. Ranganath C, Minzenberg MJ, Ragland JD.The cognitive neuroscience of memory function and dysfunction in 
schizophrenia. Biol Psychiatry. 2008 Jul 1;64(1):18-25. Epub 2008 May 21.

31. Leeson VC, Robbins TW, Franklin C, Harrison M, Harrison I, Ron MA, et al. Dissociation of long-term verbal memory and fronto-executive impairment in first-episode psychosis. Psychol Med. 2009 Nov;39(11):1799808. Epub 2009 May 7.

32. Fusar-Poli P, Perez J, Broome M, Borgwardt S, Placentino A, Caverzasi E, et al. Neurofunctional correlates of vulnerability to psychosis: a systematic review and meta-analysis. Neurosci Biobehav Rev. 2007;31(4):465-84. Epub 2007 Jan 12.

33. Dickinson D, Ramsey ME, Gold JM. Overlooking the obvious: a meta-analytic comparison of digit symbol coding tasks and other cognitive measures in schizophrenia.Arch Gen Psychiatry. 2007 May;64(5):532-42.

34. González-Blanch C, Rodríguez-Sánchez JM, PérezIglesias R, Pardo-García G, Martínez-García O, VázquezBarquero JL, et al. First-episode schizophrenia patients neuropsychologically within the normal limits: evidence of deterioration in speed of processing. Schizophr Res. 2010 Jun;119(1-3):18-26. Epub 2010 Mar 23.

35. Hartman M, Steketee MC, Silva S, Lanning K, McCann H. Working memory and schizophrenia: evidence for slowed encoding. Schizophr Res. 2003 Feb 1;59(2-3):99-113.
36. Rodríguez-Sánchez JM, Crespo-Facorro B, GonzálezBlanch C, Perez-Iglesias R, Vázquez-Barquero JL; PAFIP Group Study. Cognitive dysfunction in first-episode psychosis: the processing speed hypothesis. $\mathrm{Br} \mathrm{J}$ Psychiatry Suppl. 2007 Dec;51:s107-10.

37. Badcock JC, Dragoviç M, Waters FA, Jablensky A. Dimensions of intelligence in schizophrenia: evidence from patients with preserved, deteriorated and compromised intellect. J Psychiatr Res. 2005 Jan;39(1):11-9.

38. Holthausen EA, Wiersma D, Sitskoorn MM, Dingemans PM, Schene AH, Van den Bosch RJ. Long-term memory deficits in schizophrenia: primary or secondary dysfunction? Neuropsychology. 2003 Oct;17(4):539-47.

39. Niendam TA, Bearden CE, Rosso IM, Sanchez LE, Hadley T, Nuechterlein KH, et al. A prospective study of childhood neurocognitive functioning in schizo-phrenic patients and their siblings. Am J Psychiatry. 2003 Nov;160(11):2060-2.

40. Leeson VC, Barnes TR, Harrison M, Matheson E, Harrison I, Mutsatsa SH, et al. The relationship between IQ, memory, executive function, and processing speed in recent-onset psychosis: 1-year stability and clinical outcome. Schizophr Bull. 2010 Mar;36(2):400-9. Epub 2008 Aug 4. 